\title{
MENTAL HEALTH ISSUES IN CAREGIVERS OF CANCER PATIENTS
}

\author{
Anila Mukhtar* \\ Anila Amber Malik ${ }^{* *}$ \\ Ayesha Rasool ${ }^{* * *}$
}

\begin{abstract}
Cancer, a terminal illness, has long been a focus of attention due to its fatal nature. Its diagnosis results in distress not only to the patients and physicians, but also the families and caregivers involved. This distress is of a multifaceted nature, including psychological, financial and physical distress. The present paper aims to explore mental health issues that specifically influence cancer patients' caregivers. However, in a country like Pakistan literature is not very rich regarding the mentioned issue. Therefore, giving rise to the immense need to create awareness concerning this pressing issue. In this paper, an effort has been made to identify the types of mental health problems and variables which could potentially affect the mental health of a cancer patient caregiver. An analysis on the type of issues and associated determining variables reveals various patients' variable, situational variable or the caregiver's own personality which makes them susceptible to poor mental health. Furthermore, the paper tries to answer the different question regarding the prevalence rate, current status of, and awareness on the above mentioned aspect. In the end some possible recommendations have also been made in the context of the mental health issues regarding the caregivers of cancer patients discussed in the paper.
\end{abstract}

Keywords: Caregivers, cancer patients, mental health

\section{Introduction}

There are a number of fatal diseases that affect the mental and physical life of a person and the lives of those related to them. One of these many diseases is cancer. Cancer is a general term used for a group of diseases that occur when there is an uncontrolled growth of abnormal cells in one or more organs or tissues of the body. Cancer is a serious disease and is one of the leading causes of death globally. ${ }^{1}$ This definition merely covers the physical or physiological understanding of cancer. However, through the perception of a human, it is not merely a reaction taking place inside a volcano; it is more than just an eruption leading to death; it is the aftermath that follows, and leaves all present nearby affected drastically. According to "World Cancer Death Clock", the annual rate of deaths caused by cancer alone is 6202080. As we go through literature, we see a psychological picture as well, attributed to the fact that cancer is stigmatized as

\footnotetext{
* Anila Mukhtar, Assistant Professor, Department of Psychology, University of Karachi, Karachi

*** Anila Amber Malik, Ph.D. Professor, Department of Psychology, University of Karachi, Karachi

*** Ayesha Rasool, Department of Psychology, University of Karachi, Karachi

1 "Cancer: Definition", World Health Organization, 2014

http://www.who.int/mediacentre/factsheets/fs297/en/index.html (accessed November 12, 2011)
} 
a life threatening illness. It places the patient at an extreme pressure point leading to intense fear of expected death, mental shock, helplessness, and self-pity.

It is important to understand the role and status of a caregiver in a cancer patient's life as the diagnosis of cancer leaves its mark in every facet of his life, such as, emotional, psychological, personal and daily regimes. According to health dictionary a caregiver is an individual who provides care to those who need supervision or assistance in illness or disability. This may be carried out at home, in a hospital, or in an institution. Although, professionally caregivers include trained medical, nursing, and other health personnel, the concept has now broadened due to home care facilities to include parents, spouses, or other family members, friends, members of the clergy, teachers, social workers, fellow patients, etc. ${ }^{2}$

With the advancing circle of caregivers, categorization into formal and informal caregivers must be clarified. One of the primary differences between the two is of the array of tasks and responsibilities. This article chiefly focuses on informal caregivers and their mental health issues, due to them being physically and psychologically associated with the patients. Informal caregivers, also referred to as family caregivers, provide a complex array of support tasks that extend across physical, psychological, spiritual, and emotional domains. ${ }^{3}$

The diagnosis of cancer may cause the care givers of cancer patients' intense emotional responses like shock, doubt, anxiety, and depression. ${ }^{4,5}$ Hence, other than the mental health and its issues of cancer patients, the response toward the illness and mental health of the significant others of the patients are also important.

Family caregivers are an important element of supportive therapy for intense illnesses like cancer. An important component that must be considered when determining psychological well-being of an individual is the age and gender to understand the mechanism of impact. According to a review of present literature, there is a consistency between Western and Eastern culture in terms of age and gender variables as the caregivers are mostly females and between the age group of 50 to $55 .{ }^{6,7} \mathrm{~A}$ study conducted in the Agha Khan Hospital as a tertiary care hospital, supports these findings

\footnotetext{
${ }^{2}$ Arin Sorensen, ed; Definition of Caregiver Online Health Dictionary Corp. Department of Medical Web Publishing 2005. Editorial Liber S.L, Company registered with No. 10473 Registre de ocietats Mercantils del M.I. Govern, www.healthdictionary.info (accessed on November 10, 2011)

${ }^{3}$ Honea N. J, Brintnall R, Given B, et al.: Putting Evidence into Practice: Nursing Assessment and Interventions to Reduce Family Caregiver Strain and Burden. Clin J Oncol Nurs 12 (3): 507-16, 2008.

${ }^{4}$ Given CW, et. al. The Caregiver Reaction Assessment (CRA) for caregivers to persons with chronic physical and mental impairment. Res Nurs Health (1992); 15: 271-83

${ }^{5}$ Kurtz M.E. et.al. Relationship of Caregiver Reactions and Depression to Cancer Patients' Symptoms, Functional States and Depression: A Longitudinal View. Soc Sci Med 1995; 40: 837-46

${ }^{6}$ Alecxih LMB, Zeruld S, Olearczyk B: Characteristics of Caregivers Based on the Survey of Income and Program Participation. National Family Caregiver Support Program: Selected Issue Briefs. (Washington, DC: U.S. Department of Health and Human Services, Administration on Aging, 2001). (accessed June 16, 2011)

${ }^{7}$ Sherwood PR, Given BA, Given CW, et al.: Predictors of Distress in Caregivers of Persons with A Primary Malignant Brain Tumor. Res Nurs Health 29 (2): 105-20, 2006. [PUBMED Abstract]
} 
by stating a total of $83 \%$ of female caregivers, ranging between the ages of 51-55 out of the 200 caregivers of cancer patients. ${ }^{8}$

The natures of tasks the care givers provide require intensive physical and psychological involvement. From managing the medications, medical files and finding appointments, to taking the patients to the hospital, maintaining their diet, and helping them in daily hygiene routines. Sometimes, they have to perform a nursing role, and in case of old age or extreme weakness of patient, the physical support is palpable. More technical jobs like intravenous injections, maintaining oxygenation of patients at home and wound care are few stressful but routine jobs. ${ }^{9}$ More specifically they may have to integrate new medical information, illness related terminology, and enter a new treatment setting altogether. $^{10}$

As indicated earlier that the caregivers not only give physical support, but are also responsible to provide psychological help like emotional and social support, by giving them ultimate relief from the worries and pains, by incorporating life style changes, ${ }^{11}$ making them motivated and mentally prepared for chemotherapy, giving them mental relaxation, in short doing the best effort to make whole scenario as comfortable as they can, inclining them toward health achievement as compared to disorder.

This multifaceted role of a caregiver signifies a bare truth that as the role demands go higher in terms of responsibility and sensitive involvement, the higher the threat to mental health of the caregiver, despite the differences of the conditions and stage of the cancer.

The magnitude of impact that cancer has, leads towards the impending question of its awareness and treatment. Although abundant in research field, it lacks significant reporting in literature about the mental health issues of caregivers and cancer patients. Despite the caregivers being a "focus of attention" recently, there have been certain limitations as to the main questions and underlying dynamics to be uncovered. For instance, the studies conducted have analyzed the psychological stress, anxieties, depression with reference to economic challenges, age of the patients, and stage of the disorder, but there is scarcity of the strategic domain.

Hence, it is important to investigate the actual issues faced by the caregivers and their coping strategies. Determining the role of health care authorities and state is the need of the hour.

\footnotetext{
${ }^{8}$ Yousafzai A. et.al. Caregivers' Stress Of Cancer Patients In A Tertiary Care Hospital. Journal of Postgraduate Medical Institute, 22 (2008): 62-65

${ }^{9}$ Zarit SH. Family Care and Burden at the End of Life. Can Med Assoc J 2004; 12: 1811-2

${ }^{10}$ Lajchen M: Role of Family Caregivers in Cancer Pain Management. In: Bruera ED, Portenoy RK, eds.: Cancer Pain: Assessment and Management. 2nd ed. (New York, NY: Cambridge University Press, 2009) 597607.

${ }^{11}$ Given BA; Given, CW; Kozachik S, "Family Home Care for Individuals with Cancer". A Cancer Journal for Clinicians, 51 (July/August 2001): 213-231. DOI: 10.3322/canjclin.51.4.213 Article first published online: 31 Dec 2008
} 


\section{Objective}

The present paper is aimed to explore the issues which have been addressed regarding mental health of caregivers of cancer patients, the potential variables, and to find the gaps and the ignorant areas in these contexts. Furthermore, a debate on its importance is also a significant part of the paper.

\section{Analysis}

With the advancements and change in the treatment plan, new development, and technology in the strategic customs of healing cancer, a sudden shift in the role of caregivers of cancer patients has occurred. Outlook on the therapy sessions (chemotherapy or radio therapy) shifted altogether, being less time consuming and efficient. Caregiving responsibility of formal caregivers has increased in contrast to informal caregivers with the rise of outpatient dealing. As far as the mental health of the caregivers is concerned, the informal caregivers are at a higher risk of being affected as compared to the formal caregivers. The basic reason for this higher risk is emotional involvement with the patient, which informal caregivers already possess. Or this is developed during caregiving process. The majority of informal caregivers are middle aged parents, especially mothers, spouses- mostly wife, siblings, daughters and sometimes friends, teachers, volunteers or relatives etc. Involvement may vary with the status of relationship. As far as the gender is concerned, all Eastern and Western studies are consistent that females are the usual caregivers. Considering age, gender and relationship with patient as primary determinants of caregiving characteristics it was found that caregivers with these characteristics could be at a higher risk because of the level of the involvement they have. Studies in both Eastern and Western cultures show that mothers are supposed to be most sensitive and sympathetic for their children. They provide emotional support by completely owning the pains and feelings of the patient. Hence, they are more vulnerable to psychological burden and stress as compared to others. This theoretical assumption is consistent with the results of another study done which states that majority of care givers are females (house wives and mothers of the patient) and most female caregivers show significantly higher score on General Health questionnaire. ${ }^{11}$ Another study reported that women had significantly higher prevalence of Hospital Anxiety Depression Scale (HADS) depression compared to males. ${ }^{12}$

An interesting fact under the collectivistic cultural context is that some studies showed an entirely different picture regarding the effects of close relationship. Caregivers having an emotional involvement with the patient consider caregiving a satisfying experience instead of a stressful experience.

Researchers have successfully identified the mental health issues like stress, anxiety and depression among caregivers especially in the primary caregivers. ${ }^{13}$ Most of the research based issues can be categorized into three major categories namely;

\footnotetext{
${ }^{12}$ Grov E. K, et. al. Anxiety, Depression, and Quality of Life in Caregivers of Patients with Cancer in Late Palliative Phase. Ann Oncol 2005 16(7):1185- 91.

${ }^{13}$ Nijboer, C. et.al. The role of social and psychologic resources in caregiving of cancer patients. Cancer, 91(2001): 1029-1039. doi: 10.1002/1097-0142(20010301)91:5<1029::AID-CNCR1094>3.0.CO;2-1
} 
i) conditions in which the caregiver provides help,

ii) patient's characteristics,

iii) caregiver's characteristics.

Firstly, considering the conditions in which caregivers provide help, majority are dependent on the external environmental status like lower socioeconomic status, lack of family support, duration of the cancer, poor prognosis and more frequent changes in care giving activities. All of these factors negatively affect mental wellbeing of the caregiver. ${ }^{14}$ A more culturally specific factor which plays a vital role in a developing country like Pakistan is that of the low economic conditions which influence every arena of life, be it mental, physical, educational, or political, hence affecting the health care system and financial support for the patient tremendously.

Second, as far as the patient's own characteristics are concerned age, gender, patient's high dependency and mental health are mainly focused. ${ }^{15}$ It has been found that caring for an elderly patient creates a negative impact on caregiver's self-efficacy and develops a sense of burden. ${ }^{15}$

Thirdly, the most crucial of all is the caregiver's characteristics, which should be the focus of research in this field, but unfortunately has been sidelined until recently. However, few researchers have focused on educational level, socioeconomic status, selfefficacy, physical strength, self-esteem, neuroticism, optimism, and mastery of caregiver, as potential variables. The research literature on these variables shows an inconsistent classification. Self-esteem and physical strength have been found to be a predictor of care giving quality.

A point to question is that if self-efficacy, neuroticism, optimism, and mastery are basically personality variables, then why were they studied after analyzing them in a period of care giving activities, instead of studying these characteristics as a prerequisite of care giving activities? If care giving is an ongoing process and it is, especially for cancer patients suffering a chronic condition, then the caregiver's self-esteem level before the onset of informal care-giving is of importance. It can also be interpreted that those researchers merely found out the effect of care giving on certain personality traits but not the effect of personality trait on quality and potential of caregiving. The importance of traits can be analyzed through the fact that personality features may directly or indirectly affect our behavior or at times play as a mediator variable. For instance, people in the same situations, but with different personalities behave differently. It is because personality is a combination of genotypic and phenotypic behavior, which develops through a person's perception, memories, strengths, and weaknesses. Hence, reaction in the same situation would be different for different people. This notion is also consistent with the other researchers, as Nijbore (2001) argues "personal perceptions of the situation play a major role in explaining variations

\footnotetext{
${ }^{14}$ Nijboer C. et.al. Cancer And Caregiving: The Impact On The Caregiver's Health. .Psycho-Oncology 7 (1998): 3-13

${ }^{15}$ Nijboer C. et.al. Determinants of caregiving experiences and mental health of partners of cancer patients. Cancer, 86 (1999): 577-588. doi:10.1002/(SICI)1097-0142(19990815)86:4<577::AID-CNCR6>3.0.CO;2-S
} 
in caregiver outcome". ${ }^{16}$ Therefore, objectively analyzing personality features of the caregiver is important to judge which personalities are suitable to perform the role of informal caregiver and which are not. Unfortunately the research literature is scarce in this regard as Nijbore discussed that "the consequences for the caregivers still are unclear. To differentiate the impact of caregiving, and to identify caregivers at risk, a thorough investigation of factors influencing caregiving consequences appears warranted". ${ }^{17}$

Although characteristics of care-givers play an important determinant in increasing the quality of facilitation, more objective factual information may also help in increasing care. In order to obtain this factual data state managed specific health related databases are required to keep record information related to any particular disorder. This provides help for further investments whether educational, economic or interventions in policy making etc. Literature is scarce regarding the facts and figures of cancer patients. 'Cancer registration' is a process of continuing, systematic collection of data on the occurrence and characteristics of reportable neoplasm." A cancer registry attempts to collect, store, analyze, and interpret data on persons with cancer. A population-based registry records all new cases in a defined population with emphasis on epidemiological research, and evaluation of health services for prevention, diagnosis, and treatment of the disease.

This leads us to an alarming question that is there any specific cancer registry available in Pakistan and if yes then at what level is it functioning? The possible answer is that there is no such data base available regarding cancer which can assure the current status of Pakistani population afflicted by cancer. The only systematic registry in Pakistan is maintained by Karachi Cancer Registry (KCR), based on the reported cases in district of Karachi South. However, KCR has its limitations; the data is based on just one district of Karachi. Nevertheless this data is taken as a sample for population of Pakistan due to its inclusion of ethnic and socioeconomic diversity. Although it is a useful document but not at par with the current impeding need.

Now the question arises why it is not possible to have data like KCR for the whole of Pakistan? The answer being the same sad truth which serves many of our other state problems also, lack of resources. It is not possible to have a database like KCR or another established by Western countries, because it requires highly modified and latest technology to collect, store, and maintain the data from all over Pakistan. According to certain evaluators, in a country like Pakistan, this type of registry needs at least two decades to establish, due to lack of mandatory resources and technology. ${ }^{18}$

Continuing with the discussion of care givers, an important element is their mental health and stability. And with it arises the immense need of psychoeducation and awareness programs for the caregivers, in order to help them seek mental health support if and when needed. Or help in determining at which point they should ask for the

\footnotetext{
${ }^{16}$ Nijboer, C. et.al. Op.cit.

${ }^{17}$ Ibid.

${ }^{18}$ Bhurgri Y. et. al. Large-scale pathology-based cancer data a reflection of population-based cancer data. Pathol Oncol Res 2002;8:62-7
} 
professional help? What is the prevalence of caregivers consulting professionals? Do they feel the need to discuss it with others or not?

After an intensive literature review it can be determined that there is substantial scarcity of data particularly on the mental health of caregivers, indicating the alarming need for attention on this topic by the Health Care Service providers and the responsible authorities like Health Ministry. Few researches are at an initial stage to answer the burning question of mental health problems of caregivers of cancer patients. The further questions are related to caregiver's awareness and consultation level, additionally, an Oncologist's role in identifying the maladjustments of caregivers. Few researchers have shown, with a few exceptions that caregivers are more worried about the health facilities given to their patients than their own comfort thereby not consulting professionals for their own misery. The reason might be financial constraints, mental preoccupation with the caregiving activities, lack of energy or time and negligence, according to a report of CA. ${ }^{19}$ Caregivers do provide love for patients but forgo their own mental health. Findings of an intensive clinical interview indicated that almost half of the caregivers do not take mental health services, though they have diagnoses for mental illnesses.

The finding of a research conducted in 2005 , being that "out of 200 caregivers twentysix $(13 \%)$ of the caregivers met the diagnostic criteria for one or more psychiatric disorders. Twenty-one (80.8\%) of them said they had discussed a mental health concern with a health care provider before their loved one was diagnosed with cancer, but only 12 of them $(46.2 \%)$ had sought professional help with their mental health after their loved one's diagnosis". ${ }^{20}$ There is no such data available to show the prevalence of caregiver's usage of mental health services in Pakistan.

The alarming state of mental health stability of caregiver's of cancer patients raises a critical consideration, that if caregivers are not caring for themselves and scarcely consult with psychologists or other professionals, then the reason might be lack of available services. If we look at the services available specifically for the caregivers in developed countries, it is evident that they, too, have certain shortcomings and need a lot of improvement. And that need, will be intense in the underdeveloped and developing countries like Pakistan. The situation is much compelling when we analyze the available resources even for cancer patients themselves. According to advocacy policy of WHO, more than $70 \%$ of all cancer deaths occur in low-middle-income countries, where resources available for prevention, diagnosis, and treatment of cancer are limited or nonexistent. ${ }^{21}$ But as discussed earlier, the developed nations are more aware of the need of solving mental health issues and are placing effort to develop systematic programs to help caregivers. For example, there are certain organizations such as the Family Caregiver Alliance, who are working specifically for caregiver's health, especially those

\footnotetext{
${ }^{19}$ Family Caregivers Often Neglect their Own Mental Health. CA: A Cancer Journal for Clinicians, 2006; 56: 5-6. doi: 10.3322/canjclin.56.1.5

${ }^{20}$ Vanderwerker LC, Laff RE, Kadan-Lottick NS, et al: Psychiatric disorders and mental health service use among caregivers of advanced cancer patients. Journal of Clinical Oncology; 2005: 23:6899-6907 Target

${ }^{21}$ World Health Organization,WHO. "Cancer Control Knowledge Into Action: Policy And Advocacy" WHO Guide For Effective Program. 2008: module 6.
} 
who are with chronically ill patients. They help them financially, giving awareness and training programs, and carrying out researches in this regard for a better service. ${ }^{22}$

An interesting fact is that most interventions identified in literature are introduced by the researchers in different articles as a project. This means that these interventions are not particularly available for the masses; rather they have just been suggested and still need to be implemented by State, Community Health Services, or Medical Health Units.

\section{Conclusion and Recommendations}

The above mentioned discussion can be fruitful if we draw some insight provoking inferences, in order to layout some strategies to help caregivers who have mental discomforts; due to the intensive informal care they provide to patients suffering from cancer.

The general inference, which can be drawn from this discussion, is that no doubt there are many thorny barriers between the currently faced issues and their solutions, especially with reference to developing countries like Pakistan, India, etc. the point of focus should be finding solutions to this issue, for which every element of society should play its part.

Any problem which has to be solved objectively must be identified first. To identify the problem any means can be utilized be it philosophical, general, observational or other. At this stage, the presented issue needs the active role of researchers and media that can stimulate and target the policy makers, health care units, state, and last but not the least, the primary stake holders - the general population. For this, intensive research would be the responsibility of different medical and social sciences institutes, while the awareness campaigns and programs could be handled through media, community, and organizations of social welfare, educationists, NGOs and public figures like artists, sports figure.

This phase should be followed by intensive and efficient feedback through active communication channels like cell phones, text, emails, and social communication networks. These steps can be followed by maintenance of registry data base at each level, which would lead to better policy making and fund raising programs, whether through state, industries or donors. By having complete information and knowledge of strength and weaknesses efficient health care systems can be established.

For a more systematic planning inspirations can also be taken from the available documents delivered from different organizations like WHO, UNICEF, Human Right's Commission etc. ${ }^{23}$

\footnotetext{
${ }^{22}$ Family Care Giver Alliance. (FCA). National Centre On Caregiving. San Francisco. file:///D:/FCA\%20\%20Family\%20Caregiver\%20Alliance\%20Home.htm

${ }^{23}$ World Health Organization,WHO. "Cancer Control Knowledge Into Action: Policy And Advocacy" WHO guide for effective program. 2008: module 6
} 


\section{Bibliography}

Alecxih LMB, Zeruld S, Olearczyk B: Characteristics of Caregivers Based on the Survey of Income and Program Participation. National Family Caregiver Support Program: Selected Issue Briefs. Washington, DC: U.S. Department of Health and Human Services, Administration on Aging, 2001.

Arin Sorensen, ed; Definition of Caregiver Online Health Dictionary Corp. Department of Medical Web Publishing 2005. Editorial Liber S.L, Company registered with No. 10473 Registre de ocietats

Bhurgri Y, Hasan SH, Pervez S, et al Large-scale pathology-based cancer data a reflection of population-based cancer data. Pathol Oncol Res 2002

“Cancer: Definition”, World Health Organization, 2014

http://www.who.int/mediacentre/factsheets/fs297/en/index.html

Family Care Giver Alliance. (FCA). National Centre On Caregiving. San Francisco. file:///D:/FCA\%20\%20Family\%20Caregiver\%20Alliance\%20Home.htm

Family Caregivers Often Neglect their Own Mental Health. CA: A Cancer Journal for Clinicians, 2006

Given BA; Given, CW; Kozachik S, "Family Home Care for Individuals with Cancer". A Cancer Journal for Clinicians, 51 (July/August 2001): 213-231. DOI: 10.3322/canjclin.51.4.213 Article first published online, 2008

Given CW, Given B, Stommel M, Collins C, King S, Franklin S. The Caregiver Reaction Assessment (CRA) for caregivers to persons with chronic physical and mental impairment. Res Nurs Health 1992

Grov E. K, Dahl AA, Moum T, Fossa SD. Anxiety, Depression, and Quality of Life in Caregivers of Patients with Cancer in Late Palliative Phase. Ann Oncol 2005.

Honea N. J, Brintnall R, Given B, et al.: Putting Evidence into Practice: Nursing Assessment and Interventions to Reduce Family Caregiver Strain and Burden. Clin J Oncol Nurs 12 (3): 2008.

Kurtz ME, Kurtz JC, Given CW, Given B. Relationship of Caregiver Reactions and Depression to Cancer Patients’ Symptoms, Functional States and Depression: A Longitudinal View. Soc Sci Med 1995

Lajchen M: Role of Family Caregivers in Cancer Pain Management. In: Bruera ED, Portenoy RK, eds.: Cancer Pain: Assessment and Management. 2nd ed. (New York, NY: Cambridge University Press, 2009).

Mercantils del M.I. Govern, www.healthdictionary.info

Nijboer C, Tempelaar R, Sanderman R, Trimstra M, Spruijt R. J and Vandenbos G.A.M. Cancer And Caregiving: The Impact On The Caregiver's Health. .Psycho-Oncology 7 (1998)

Nijboer C, Tempelaar R, Sanderman R, Trimstra M, Spruijt R. J and Vandenbos G.A.M Determinants of caregiving experiences and mental health of partners of cancer patients. Cancer, 86 (1999) doi:10.1002/(SICI)1097-0142(19990815)86:4<577::AID-CNCR6>3.0.CO;2-S

Nijboer, C., Tempelaar, R., Triemstra, M., van den Bos, G. A. M. and Sanderman, R. The role of social and psychologic resources in caregiving of cancer patients. Cancer, 91(2001): 1029-1039. doi: 10.1002/10970142(20010301)91:5<1029::AID-CNCR1094>3.0.CO;2-1

Sherwood PR, Given BA, Given CW, et al.: Predictors of Distress in Caregivers of Persons with A Primary Malignant Brain Tumor. Res Nurs Health 29 (2): 105-20, 2006. [PUBMED Abstract] 
Vanderwerker LC, Laff RE, Kadan-Lottick NS, et al: Psychiatric disorders and mental health service use among caregivers of advanced cancer patients. Journal of Clinical Oncology; 2005

World Health Organization,WHO. "Cancer Control Knowledge Into Action: Policy And Advocacy” WHO Guide For Effective Program. 2008: module 6.

Yousafzai A, Bhutto N, Ahmer S, Siddiqi M. N, Salamat S Caregivers' Stress Of Cancer Patients In A Tertiary Care Hospital. Journal of Postgraduate Medical Institute, 22 (2008)

Zarit SH. Family Care and Burden at the End of Life. Can Med Assoc J 2004 